\title{
The Perioperative Effect of Increased Body Mass Index on Peripheral Nerve Blockade: an Analysis of 528 Ultrasound Guided Interscalene Blocks
}

\author{
Kristopher Schroeder ${ }^{1}$, Adin-Cristian Andrei ${ }^{2}$, Meghan J Furlong ${ }^{3}$, Melanie J Donnelly ${ }^{1}$, \\ Seungbong Han ${ }^{4}$, Aimee M Becker ${ }^{1}$
}

\begin{abstract}
Summary: Schroeder K, Andrei AC, Furlong MJ, Donnelly MJ, Han S, Becker AM - The Perioperative Effect of Increased Body Mass Index on Peripheral Nerve Blockade: an Analysis of 528 Ultrasound Guided Interscalene Blocks.
\end{abstract}

Background and objectives: Obese patients can pose a unique perioperative anesthetic challenge, making regional anesthetic techniques an intriguing means of providing analgesia for this population. Ultrasound guidance has been touted recently as being beneficial for this population in which surface landmarks can become obscured. In this study, the effect of increased Body Mass Index (BMI) on ultrasound guided interscalene peripheral nerve blockade is investigated.

Material and methods: This study is a retrospective review of 528 consecutive patients who received preoperative ultrasound-guided interscalene nerve blocks at the University of Wisconsin Hospital and Clinics. We examined the association between BMl and the following parameters: time required for block placement; presence of Postoperative Nausea and Vomiting (PONV); postoperative Post Anesthesia Care Unit (PACU) pain scores; volume of local anesthetic injected; acute complications; and opioid administration preoperatively, intraoperatively, and postoperatively. Univariate and multivariate least squares and logistic regression models were used.

Results: An elevated BMI was associated with an increased: time required for block placement ( $p$-value $=0.025)$, intraoperative fentanyl administration ( $p$-value $<0.001$ ), peak PACU pain scores ( $p$-value $<0.001$ ), PACU opioid administration ( $p$-value $<0.001$ ), PACU oral opioid administration ( $p$-value $<0.001$ ), total PACU opioid administration ( $p$-value $<0.001)$ and incidence of PACU nausea $(p$-value $=0.025)$

Conclusions: Ultrasound guided interscalene nerve blocks for perioperative analgesia can be safely and effectively performed in the obese patient but they may be more difficult to perform and analgesia may not be as complete.

Keywords: Nerve Block; Body Mass Index; Ultrasonography.

\section{INTRODUCTION}

As of 2005, the World Health Organization (WHO) estimated that 1.6 billion adults were overweight with more than 400 million being obese. By 2015 , WHO predicts that 2.3 billion adults will be overweight with 700 million obese. Body Mass Index (BMI) is calculated by dividing the body weight in kilograms by the square of the height in meters and has been divided into five categories: $<25 \mathrm{~kg} \cdot \mathrm{m}^{-2}=$ normal, $25-30 \mathrm{~kg} \cdot \mathrm{m}^{-2}=$ overweight, $>30 \mathrm{~kg} \cdot \mathrm{m}^{-2}=$ obesity, $>35 \mathrm{~kg} \cdot \mathrm{m}^{-2}=$ morbid obe-

Received from University of Wisconsin School of Medicine and Public Health, USA.

1. MD; Assistant Professor, Department of Anesthesiology, University of Wisconsin School of Medicine and Public Health, Madison, Wisconsin, USA

2. PhD; Senior Biostatistician, Northwestern University

3. MD; Resident Physician, Department of Anesthesiology, University of Colorado, Denver

Colorado, USA

4. PhD; Statistician, Asan Medical Center, South Korea

Submitted on March 6, 2011

Approved on May 19, 2011.

Correspondence to:

Kristopher Schroeder MD

University of Wisconsin, School of Medicine and Public Health

Department of Anesthesiology B6/319 CSC

600 Highland Avenue Madison, WI 53792 USA

E-mail:kmschro1@wisc.edu sity, $>55 \mathrm{~kg} \cdot \mathrm{m}^{-2}=$ super morbid obesity ${ }^{1}$. Obese individuals undergoing surgery pose specific challenges for anesthetic management. People with increased BMI are at higher risk for intraoperative and postoperative pulmonary atelectasis ${ }^{2}$. They also often suffer from obstructive sleep apnea (OSA) predisposing them to desaturation, hypoxemia and difficult intubation ${ }^{3,4}$. Additionally, overweight and obese people are more likely to have underlying cardiovascular diseases such as hypertension and congestive heart failure than nonobese ${ }^{5}$.

Regional anesthesia techniques have been gaining popularity in the obese and normal weight patient populations for many reasons. Most noteworthy among these reasons are that regional anesthesia is associated with improved postoperative pain control. Reduced pain results in decreased opioid administration. A benefit of this is less antiemetic use secondary to decreased postoperative nausea and vomiting (PONV), and decreased time in post anesthesia care units (PACU) leading to increased patient satisfaction ${ }^{6,7}$. The combination of increasing numbers of obese patients with their unique underlying medical problems and the growing popularity of regional anesthesia makes the use of regional anesthetic techniques in the obese population a topic that warrants further investigation. 
Few studies have thoroughly investigated the impact of obesity on the success rate of regional anesthesia. Thus, in most of the studies either paresthesia or nerve stimulator techniques for nerve localization have been used. Ultrasound guidance for interscalene brachial plexus nerve blocks (ISBN) in particular has been demonstrated to result in a high success rate and superior results when compared to nerve stimulation alone ${ }^{8,9}$. Ultrasound guidance is an attractive tool in overweight and obese patients as surface landmarks are often obscured and difficult to palpate. A small study of 70 patients evaluated the impact of obesity on ultrasound guided interscalene blocks and reported that being overweight was only associated with increased time for block placement. This study could not detect an increase in block failure rate in the obese population ${ }^{10}$. The goal of our study was to analyze a large group of patients presenting for shoulder arthroscopy using an interscalene block for postoperative analgesia to determine if increased BMI impacts block success via a variety of clinical parameters.

\section{MATERIALS AND METHODS}

Following approval by the Institutional Review Board at the University of Wisconsin, Madison, the medical records of 605 patients who underwent preoperative interscalene nerve blockade for shoulder surgery between the dates of 12/7/2006 and 9/16/2008 were reviewed. Minors, security patients, and those undergoing arthroplasty or hemiarthroplasty were excluded from analysis resulting in a total of 529 patients.

All patients underwent preoperative ultrasound guided single-injection interscalene nerve blocks in a room dedicated to performing regional anesthesia. Blocks were performed by mid to senior level anesthesia residents under the supervision of four faculty anesthesiologists trained in regional anesthesia. Standard monitors were applied and patients were sedated with midazolam and/or fentanyl. Ultrasound guidance was then used to localize the brachial plexus in the interscalene groove. A stimulating needle was then inserted either "in-plane" or "out-of-plane" to the ultrasound beam. Current was applied to the needle and an attempt was made to obtain either a biceps or deltoid contraction at a current of approximately $0.5 \mathrm{~mA}$ prior to injecting local anesthetic. Local anesthetic was injected and the needle redirected until good spread of local anesthetic was visualized around the brachial plexus. No formal testing of motor or sensory blockade took place following block placement. Patients were then transported to the operating room where they underwent general anesthesia with endotracheal intubation for their surgical procedure. Intraoperative opioids and antiemetics were provided at the discretion of the in-room anesthesia personnel. Following the surgical procedure, patients were transported to the PACU.

Patient data extracted from the medical record included age, gender, ASA score, date of surgery, surgical procedure, height, weight, preoperative opioid use, time for block placement, medications required for block placement, volume of local anesthetic injected for block, intraoperative fentanyl use, intraoperative and postoperative hydromorphone use, postoperative intravenous (IV) and oral (PO) opioid administration, immediate and peak PACU postoperative pain, presence of postoperative PACU nausea, and presence of acute complications. Pain scores were patient reported subjective numerical rating scores (NRS) between zero and 10 where zero represented no pain and 10 represented the worst pain imaginable. Acute complications included seizure, need for immediate postoperative intubation, unanticipated inpatient admission, or note of a failed block in the PACU notes.

In our statistical analyses, univariate and multivariate least squares and logistic regression models were constructed to describe how BMI is associated with: time required for the block placement, amount of fentanyl administered for block placement, intraoperative fentanyl administration, intraoperative hydromorphone administration, postoperative hydromorphone administration, total PACU opioid administration, immediate PACU postoperative pain score, peak PACU postoperative pain score, and nausea. Adjustments were made for conversion to an open procedure, age, sex, preoperative opioid consumption and ASA score.

\section{RESULTS}

Five hundred and twenty-nine ultrasound-guided interscalene nerve blocks were analyzed. One patient's height measurement was missing, hence so was the BMI. Throughout, we have used information from 528 participants. Table I summarizes demographic and other baseline characteristics, including preoperative opioid usage, ASA score and incidence of arthroscopic procedure being converted to open procedure. Table II summarizes outcome data across the different BMI categories. Table III presents univariate and multivariate least squares regression model results for the post- or intra-operative outcomes of interest. No difference in volume of local anesthetic injected was noted between groups. An increased BMI was associated with statistically significant increases in each of the following outcomes ( $p$-values in univariate and multivariate models, respectively, are shown):

(a) peak PACU pain scores (p-values: $<0.001$ and <0.001),

(b) time for ISNB placement (p-values: 0.018 and 0.025),

(c) intraoperative fentanyl administration ( $p$-values: 0.004 and $<0.001$ ),

(d) PACU opioid administration (p-values: < 0.001 and <0.001),

(e) PACU oral opioid administration ( $p$-values: 0.003 and < 0.001 ) and

(f) PACU total opioid administration (p-values: $<0.001$ and $<0.001$ ). 
Table I - Patient Characteristics $(\mathrm{N}=528)$

\begin{tabular}{|c|c|c|c|c|c|}
\hline & & $\begin{array}{l}\text { Normal } \\
(\mathrm{BMI}<25) \\
(\mathrm{N}=124)\end{array}$ & $\begin{array}{l}\text { Overweight } \\
(25 \leq \mathrm{BMl}<30) \\
(\mathrm{N}=216)\end{array}$ & $\begin{array}{l}\text { Obese } \\
(\mathrm{BMI} \geq 30) \\
(\mathrm{N}=188)\end{array}$ & $\begin{array}{l}\text { Entire } \\
\text { Cohort } \\
(\mathrm{N}=528)\end{array}$ \\
\hline \multicolumn{2}{|l|}{ Age (years) } & $40.98(16.48)$ & $48.41(14.57)$ & $48.30(13.59)$ & $46.69(15.06)$ \\
\hline \multicolumn{2}{|l|}{ BMI } & $22.90(1.62)$ & $27.37(1.38)$ & $34.76(4.22)$ & $28.94(5.42)$ \\
\hline \multicolumn{6}{|c|}{ Preoperative narcotic usage } \\
\hline & Yes & 29 & 59 & 41 & 129 \\
\hline & No & 95 & 157 & 147 & 399 \\
\hline \multicolumn{6}{|l|}{ Gender } \\
\hline & Male & $58.06 \%$ & $78.24 \%$ & $70.21 \%$ & $70.70 \%$ \\
\hline & Female & $41.94 \%$ & $21.76 \%$ & $29.79 \%$ & $29.30 \%$ \\
\hline \multicolumn{6}{|c|}{ Open surgery } \\
\hline & Yes & 21 & 20 & 22 & 63 \\
\hline & No & 103 & 196 & 166 & 465 \\
\hline
\end{tabular}

* SD: Standard Deviation.

Table II - Summary for Outcome Variables by BMI Category

\begin{tabular}{|c|c|c|c|}
\hline \multirow[t]{2}{*}{ Outcome Variable } & \multicolumn{3}{|l|}{$\begin{array}{l}\text { Mean (SD) by } \\
\text { BMI Category }\end{array}$} \\
\hline & $\begin{array}{l}\text { Normal } \\
(\mathrm{BMl}<25) \\
(\mathrm{N}=124)\end{array}$ & $\begin{array}{l}\text { Overweight } \\
(25 \leq \mathrm{BMl}<30) \\
(\mathrm{N}=216)\end{array}$ & $\begin{array}{l}\text { Obese } \\
(\mathrm{BMI} \geq 30) \\
(\mathrm{N}=188)\end{array}$ \\
\hline Immediate postoperative Pain (NRS 0-10) & $0.27(1.24)$ & $0.19(1.07)$ & $0.33(1.44)$ \\
\hline Peak postoperative Pain (NRS 0-10) & $1.10(2.07)$ & $0.90(1.91)$ & $1.84(2.63)$ \\
\hline Time required for ISNB placement (min) & $15.42(6.80)$ & $14.85(7.04)$ & $16.69(6.45)$ \\
\hline Fentanyl required for ISNB placement $(\mu \mathrm{g})$ & $44.15(23.23)$ & $41.90(24.19)$ & $39.89(28.63)$ \\
\hline Intraoperative fentanyl $(\mu \mathrm{g})$ & $67.94(46.59)$ & $73.15(50.89)$ & $83.42(50.71)$ \\
\hline Intraoperative hydromorphone $(\mu \mathrm{g})$ & $0.09(0.27)$ & $0.10(0.27)$ & $0.09(0.25)$ \\
\hline Postoperative. Hydromorphone $(\mu \mathrm{g})$ & $0.78(2.54)$ & $0.39(1.65)$ & $1.65(4.24)$ \\
\hline Other postoperative opioids $(\mathrm{mg})+$ & $0.43(1.34)$ & $0.30(2.01)$ & $0.13(0.64)$ \\
\hline Postoperative oral Opioids (mg)+ & $0.62(1.51)$ & $0.77(1.71)$ & $1.07(1.84)$ \\
\hline Total postoperative Opioids (mg)+ & $1.83(3.71)$ & $1.46(3.50)$ & $2.85(5.22)$ \\
\hline Local anesthetic volume injected for ISNB placement $(\mathrm{mL})$ & $24.96(5.32)$ & $25.49(5.19)$ & $26.2(5.16)$ \\
\hline Postoperative Nausea (\%) & 17.7 & 13.9 & 26.1 \\
\hline Complications (\%) & 0.8 & 0.5 & 2.2 \\
\hline
\end{tabular}

+ Expressed as IV morphine equivalents in $\mathrm{mg}$. 
Table III - Univariate and Multivariate Least Squares Regression Analyses Exploring the Association Between Outcome Variables on the First Column and BMI

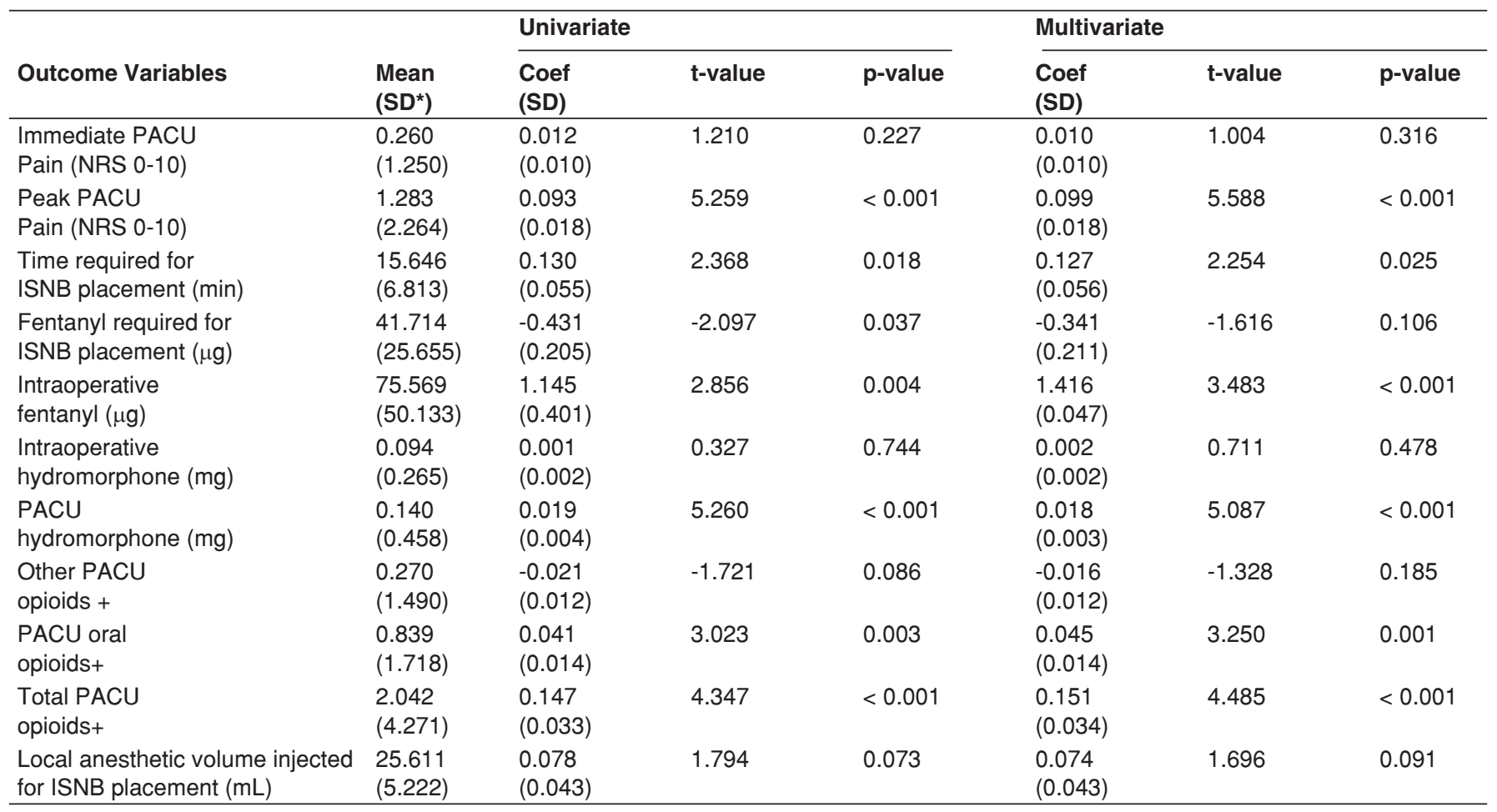

*SD: Standard Deviation.

+ Expressed as IV morphine equivalents in $\mathrm{mg}$. Multivariate model has been adjusted for age, sex, preoperative narcotic usage and ASA score.

Table IV - Univariate and Multivariate Logistic Regression Analyses Exploring the Association Between Outcome Variables on the First Column and BMI

\begin{tabular}{|c|c|c|c|c|c|c|c|c|}
\hline \multirow[b]{2}{*}{$\begin{array}{l}\text { Outcome } \\
\text { Variable }\end{array}$} & \multicolumn{2}{|l|}{ Category } & \multicolumn{3}{|c|}{ Univariate } & \multicolumn{3}{|c|}{ Multivariate } \\
\hline & $\begin{array}{l}\text { No } \\
\text { (\%) }\end{array}$ & $\begin{array}{l}\text { Yes } \\
(\%)\end{array}$ & $\begin{array}{l}\text { Coef } \\
\left(S^{*}\right)\end{array}$ & $Z$ value & $p$ value & $\begin{array}{l}\text { Coef } \\
\text { (SD) }\end{array}$ & $Z$ value & $p$ value \\
\hline Nausea & $\begin{array}{l}430 \\
(81.43 \%)\end{array}$ & $\begin{array}{l}97 \\
(18.37 \%)\end{array}$ & $\begin{array}{l}0.040 \\
(0.020)\end{array}$ & 2.038 & 0.042 & $\begin{array}{l}0.046 \\
(0.020)\end{array}$ & 2.243 & 0.025 \\
\hline Complications & $\begin{array}{l}520 \\
(98.48 \%)\end{array}$ & $\begin{array}{l}6 \\
(1.13 \%)\end{array}$ & $\begin{array}{l}0.126 \\
(0.062)\end{array}$ & 2.030 & 0.042 & $\begin{array}{l}0.110 \\
(0.067)\end{array}$ & 1.631 & 0.103 \\
\hline
\end{tabular}

*SD: Standard Deviation.

Multivariate model has been adjusted for age, sex, preoperative narcotic usage and ASA score.

In Table IV, logistic regression models adjusting for age, sex, preoperative opioid usage and ASA indicate that BMI is associated with the presence of nausea ( $p$-value $=0.025$ ). However, there is a lack of association between BMI and the presence/absence of complications ( $p$-value $=0.103$ ).

\section{DISCUSSION}

This retrospective review of 528 ultrasound-guided interscalene nerve blocks for shoulder arthroscopy demonstrated that increased BMI was associated with statistically signifi- cant increased time required for ISNB placement, increased intraoperative opioid consumption, increased peak PACU postoperative pain, increased postoperative opioid administration, increased intraoperative fentanyl administration and increased PACU nausea. This information is becoming increasingly more clinically relevant in the setting of an obesity epidemic.

An early study by Conn et al. ${ }^{11}$ that used either paresthesia or nerve stimulation to indentify the brachial plexus determined that BMI had no impact on interscalene brachial plexus block success rates. Carles et al. ${ }^{12}$ studied the brachial plexus block at the humeral canal in 1,417 patients and found that 
BMI had no impact on block success. Our results are similar to a prospective study of 9,342 blocks placed utilizing nerve stimulator technique by Cotter et al. ${ }^{13}$ In this study, all blocks completed in an ambulatory procedure center were analyzed and increased BMI and ASA IV were associated with a higher block failure rate. Nielsen et al. ${ }^{14}$ evaluated the same database as Cotter et al. ${ }^{13}$ but used a slightly different definition of block failure in addition to breaking down weight distribution even further. They were able to demonstrate that both block failure and complication rates were significantly higher in those with increased BMI. One major difference between our study and the Franco et al. ${ }^{15}$, Cotter et al. ${ }^{13}$, and Nielsen et al. ${ }^{14}$ studies is that all of our nerve blocks were placed under ultrasound guidance with peripheral nerve stimulation while theirs were done with the assistance of a nerve stimulator alone.

Franco et al. ${ }^{15}$ retrospectively reviewed the results of 2,020 supraclavicular blocks placed utilizing peripheral nerve stimulation. They determined that obesity was associated with a decrease in block placement success rate but no increase in complications. Anesthesia residents were also able to complete a lower percentage of supraclavicular nerve blocks in the obese population compared to patients of normal weight suggesting an increased level of difficulty.

Our results differ from a study by Schwemmer et al. ${ }^{10}$ that evaluated the impact of obesity on ultrasound guided interscalene blocks. This study evaluated 70 patients scheduled for shoulder surgery with a preoperative interscalene block. Their study demonstrated a small increase in the time required to indentify nerve structures in the obese patient but were unable to identify any difference in block success rates between weight classes.

Determining what constitutes a successful block is somewhat of a controversial subject. We do not routinely test for motor or sensory blockade when blocks are placed for perioperative analgesia. We have therefore evaluated other clinically relevant perioperative variables (i.e. pain scores, opioid consumption, opioid related side effects, etc.) to determine block success. In some studies, Cotter et al. ${ }^{13}$ for example, a block was considered to be a failure if a single attempt at placing a block failed to result in a complete sensory, motor, and sympathetic block. If any supplemental local anesthetic was required intraoperatively, the block was also considered a failure. In our study, all patients underwent a planned general anesthetic and the block was therefore used predominately for intraoperative opioid sparing effect and postoperative analgesia. This may explain why in our study, despite significant differences in postoperative pain scores, pain control remains quite satisfactory even in patients with an elevated BMI. In our study, secondary perioperative outcomes like increased postoperative nausea and vomiting in the obese population may offer a holistic vision of how increased pain and opioid consumption can lead to adverse clinical outcomes.
Ultrasound guidance for interscalene brachial plexus blockade has been shown to result in improved success rates, decreased number of needle passes, and faster setup times when compared to nerve stimulation ${ }^{16,17}$. Ultrasound imaging may have increased value in the obese population where surface landmarks can be difficult to either see or palpate. Unfortunately, increased adiposity also increases the depth to various targets seen on ultrasound imaging which then necessitates an increase in the angle of incidence of the needle in relationship to the ultrasound probe. This can make needle visualization more difficult and could therefore be reasonably expected to result in an increased rate of incomplete blocks if the needle tip is unable to be positioned with precise accuracy. It is unknown what impact recently introduced echogenic needles might have on the problem of difficult needle visualization in the obese population. The patients in this study had ultrasound imaging used in addition to peripheral nerve stimulation. It is unclear how this dual endpoint technique might have impacted block success rates especially in the obese population where needle tip positioning and peripheral nerve imaging can be difficult. For infraclavicular blocks, the addition of nerve stimulation to ultrasound guidance did nothing to improve block success rate or onset time ${ }^{18}$. For popliteal sciatic nerve blocks, combined ultrasound and neurostimulation guidance did result in improved block success rates ${ }^{19}$.

When using ultrasound guidance for brachial plexus blocks, the impact of obesity on normal anatomy may make interscalene blocks more difficult to perform than other approaches to the brachial plexus. When utilizing ultrasound guidance for supraclavicular blocks, the brachial plexus lies immediately lateral to the subclavian artery which is a clearly defined anatomic landmark. Despite the fact the brachial plexus can often be found at the level of the cricoid cartilage by following it cephalad from the supraclavicular position, obesity may play a larger role in increasing the difficulty of interscalene brachial plexus blocks secondary to the lack of an immediately adjacent vascular landmark.

This study has limitations that require discussion. The patients did not receive formal motor and sensory testing to evaluate the success of the interscalene nerve block. In addition, the statistically significant differences in opioid consumption may not be clinically significant especially when one considers that healthcare providers may routinely give greater doses of opioids to larger patients. Larger patients may also require greater doses of opioids to achieve similar levels of analgesia.

In summary, we believe this study demonstrates that ultrasound guided interscalene nerve blocks can be safely and effectively performed in the obese patient but that they may be more difficult to perform and analgesia may not be as complete. Therefore the incidence of opioid analgesic complications such as nausea may be increased. 
7. McCartney CJ, Brull R, Chan VW et al. - Early but no long-term benefit of regional compared with general anesthesia for ambulatory hand surgery. Anesthesiology, 2004;101(2):461-467.

8. Davis JJ, Swenson JD, Greis PE et al. - Interscalene block for postoperative analgesia using only ultrasound guidance: the outcome in 200 patients. J Clin Anesth, 2009;21:272-277.

9. Kapral S, Greher M, Huber G et al. - Ultrasonographic guidance improves the success rate of interscalene brachial plexus blockade. Reg Anesth Pain Med, 2008;33:253-258.

10. Schwemmer U, Papenfuss T, Greim C et al. - Ultrasound-guided interscalene brachial plexus anaesthesia: differences in success between patients of normal and excessive weight. Ultraschall Med, 2006;27:245-250.

11. Conn RA, Cofield RH, Byer DE et al. Interscalene block anesthesia for shoulder surgery. Clin Orthop Relat Res, 1987;216:94-98.

12. Carles M, Pulcini A, Macchi P et al. - An evaluation of the brachial plexus block at the humeral canal using a neurostimulator (1417 patients): the efficacy, safety, and predictive criteria of failure. Anesth Analg, 2001;92:197198.

13. Cotter JT, Nielsen KC, Guller U et al. - Increased body mass index and ASA physical status IV are risk factors for block failure in ambulatory surgery - an analysis of 9,342 blocks. Can J Anaesth, 2004;51(8):810-816.

14. Nielsen KC, Guller U, Steele SM et al. - Influence of obesity on surgical regional anesthesia in the ambulatory setting: an analysis of 9,038 blocks. Anesthesiology, 2005;102(1):181-187.

15. Franco CD, Gloss FJ, Voronov G et al. - Supraclavicular block in the obese population: an analysis of 2020 blocks. Anesth Analg, 2006;102:1252-1254.

16. Kapral S, Greher M, Huber G et al. - Ultrasonographic guidance improves the success rate of interscalene brachial plexus blockade. Reg Anesth Pain Med, 2008;33:253-258.

17. Liu $\mathrm{S}$, Zayas $\mathrm{V}$, Gordon $\mathrm{M}$ et al. A prospective, randomized, controlled trial comparing ultrasound versus nerve stimulator guidance for interscalene block for ambulatory shoulder surgery for postoperative neurological symptoms. Anesth Analg, 2009;109:265-271.

18. Dingermans E, Williams S, Arcand G et al. Neurostimulation in ultrasoundguided infraclavicular block: A prospective randomized trial. Anesth Analg, 2007;104:1275-1280.

19. Dufour E, Quennesson P, Van Robais A et al. - Combined ultrasound and neurostimulation guidance for popliteal sciatic nerve block: a prospective, randomized comparison with neurostimulation alone. Anesth Analg, 2008:106;1553-1558.

\section{REFERÊNCIAS / REFERENCES}

1. Bray GA. Pathophysiology of obesity. Am J Clin Nutr, 1992;55:488S-494S.

2. Eichenberger A, Proietti S, Wicky S et al. - Morbid obesity and postoperative pulmonary atelectasis: an underestimated problem. Anesth Analg, 2002;95(6):1788-1792.

3. Sinha AC - Some anesthetic aspects of morbid obesity. Curr Opin Anaesthesiol, 2009;22(3):442-446.

4. Bryson GL, Chung F, Finegan BA et al. - Patient selection in ambulatory anesthesia - an evidence-based review: part I. Can J Anaesth, 2004;51(8):768781.

5. Bryson GL, Chung F, Cox RG et al. - Patient selection in ambulatory anesthesia - an evidence-based review: part II. Can J Anaesth, 2004;51(8):782794.

6. Liu SS, Strodtbeck WM, Richman JM et al. - A comparison of regional versus general anesthesia for ambulatory anesthesia: a meta-analysis of randomized controlled trials. , 2005;101(6):1634-42. 